\title{
Load Characterization and Revenue Metering under Non-Sinusoidal and Asymmetrical Operation
}

\author{
Paolo Tenti, \\ Alessandro Costabeber, Paolo Mattavelli \\ University of Padova, Italy
}

\begin{abstract}
The paper proposes an approach to load characterization and revenue metering which accounts for the influence of supply deterioration and line impedance. It makes use of the Conservative Power Theory and aims at characterizing the load from the measurements done at the point of common coupling. Despite the inherent limitations of a single-point measurement, the proposed methodology enables evaluation of power terms which clarify the effects of reactivity, asymmetry and distortion, and attempts to depurate the power consumption accounted to the load from those terms deriving from supply non-idealities.
\end{abstract}

\section{INTRODUCTION}

The advent of smart micro-grids, characterized by distributed energy sources and pervasive use of information and communication technology (ICT), poses new and stringent requirements for power metering and load characterization. In fact, micro-grids are often fed by weak supplies, especially in case of islanded operation, where the utility is replaced by backup generators and the distributed energy sources play a major role in feeding the power required by the loads. In such instances the supply voltages can become asymmetrical and distorted, thus making the usual approaches to load characterization and revenue metering inaccurate [1-10], with possible over-penalization of blameless users or under-penalization of guilty loads.

Whatever power metering approach is taken to face such situations [11-20] it must be based on power definitions which keep their meaning even in case of distorted, asymmetrical and weak supply. This is peculiar of the Conservative Power Theory (CPT), which makes use of quantities related to power flow and energy storage, that are conservative for every network irrespective of voltage and current asymmetry and distortion $[18,19]$.

Moreover, the CPT makes possible to approach the accountability problem, i.e., assessing the load and supply responsibility on the generation of asymmetry and distortion of the voltages at the Point of Common Coupling (PCC) [21].

In [22], an accountability approach was devised, which models the load in a way that allows separation of load and supply responsibility on the generation of reactive, unbalance and distortion power measured at the point of common coupling. Here the same problem is faced by a more extensive and comprehensive formulation, which takes advantage of the sequence decomposition of voltages and

\author{
Fernando P. Marafão, \\ Helmo K. M. Paredes \\ UNESP - Univ. Estadual Paulista \\ Sorocaba, Brazil
}

currents and provides more accurate results and extended applicability to a variety of practical situations.

Since the approach relies on single-point measurements done at PCC, the corresponding load and supply modeling is necessarily affected by inaccuracy, which can be corrected by making use of additional data on actual supply and load characteristics, if available.

\section{CONSERVATIVE POWER THEORY - BASIC CONCEPTS AND DEFINITIONS}

The Conservative Power Theory, discussed in [18,19], provides power and current decomposition in the stationary regime, in a way that clearly shows the effects of voltage asymmetry and distortion, load unbalance, reactive power consumption, and non linearity. The basic CPT definitions are recalled hereafter.

Assume a generic poly-phase network under periodic operation (period $T)$, and let $\boldsymbol{u}$ and $\boldsymbol{i}$ be the voltages and currents measured at a generic port of the network (boldface symbols refer to vector quantities, while variables referring to phase $m$ are identified by subscript " $m$ "). We define:

- Unbiased integral of phase voltages

$\widehat{u}_{m}(t)=u_{m}(t)-\bar{u}_{m}$

where: $u_{m}(t)=\int_{0}^{t} u_{m}(\tau) d \tau, \quad \bar{u}_{m}=\frac{1}{T} \int_{0}^{T} u_{m}(t) d t$

- Active power (phase quantity $P_{m}$ and total amount $P$ )

$$
\left\{\begin{array}{l}
P_{m}=\left\langle u_{m}, i_{m}\right\rangle=\frac{1}{T} \int_{0}^{T} u_{m} i_{m} d t, \quad m=1 \ldots M \\
P=\langle\mathbf{u}, \mathbf{i}\rangle=\frac{1}{T} \int_{0}^{T} \sum_{m=1}^{M} u_{m} i_{m} d t=\sum_{m=1}^{M} P_{m}
\end{array}\right.
$$

- Reactive energy (phase quantity $W_{m}$ and total amount $W$ )

$$
\left\{\begin{array}{l}
W_{m}=\left\langle\hat{u}_{m}, i_{m}\right\rangle=\frac{1}{T} \int_{0}^{T} \widehat{u}_{m} i_{m} d t, \quad m=1 \ldots M \\
W=\langle\widehat{\mathbf{u}}, \mathbf{i}\rangle=\frac{1}{T} \int_{0}^{T} \sum_{m=1}^{M} \widehat{u}_{m} i_{m} d t=\sum_{m=1}^{M} W_{m}
\end{array}\right.
$$

In (2) and (3) chevron brackets represent the internal product of scalar and vector quantities.

The active power represents the rate of energy flow, while the reactive energy accounts for inductive and capacitive energy stored in the load circuit [18]. Both 
quantities are conservative for every network, irrespective of voltage and current waveforms.

Based on the above definitions, phase current $i_{m}$ can be decomposed in the following terms.

- Active phase current: $i_{a m}=\frac{P_{m}}{U_{m}^{2}} u_{m}$

- Reactive phase current: $i_{r m}=\frac{W_{m}}{\widehat{U}_{m}^{2}} \widehat{u}_{m}$

- Void phase current: $i_{v m}=i_{m}-i_{a m}-i_{r m}$

where $U_{m}$ and $\hat{U}_{m}$ are the rms values of the phase voltage and its unbiased integral.

The active and reactive phase currents are the minimum currents needed to convey, respectively, the active power and reactive energy of phase $m$, while void currents are the residual terms. Current components (4) are orthogonal.

For poly-phase systems we can further decompose the active and reactive currents into balanced and unbalanced components. The balanced active and reactive currents are defined as:

$$
\begin{aligned}
& i_{a m}^{b}=\frac{P}{\mathbf{U}^{2}} u_{m} \Rightarrow \mathbf{I}_{a}^{b}=\frac{P}{\mathbf{U}} \\
& i_{r m}^{b}=\frac{W}{\widehat{\mathbf{U}}^{2}} \hat{u}_{m} \Rightarrow \mathbf{I}_{r}^{b}=\frac{W}{\widehat{\mathbf{U}}}
\end{aligned}
$$

In (5) $P$ and $W$ are total active power and reactive energy absorbed at the measuring port, while $\mathbf{U}$ is the collective rms value of the phase voltages, defined by:

$$
\boldsymbol{U}=\|\boldsymbol{u}\|=\sqrt{\sum_{m=1}^{M} U_{m}^{2}}
$$

The same definition applies to terms $\widehat{\mathbf{U}}, \mathbf{I}_{a}^{b}, \mathbf{I}_{r}^{b}$.

The balanced active and reactive currents represent the phase currents which would be absorbed, with the same voltage supply, by a symmetrical equivalent load taking the same total active power and reactive energy of the actual load. The unbalanced active and reactive phase currents are defined by difference:

$$
i_{a m}^{u}=i_{a m}-i_{a m}^{b}, \quad i_{r m}^{u}=i_{r m}-i_{r m}^{b}
$$

All current components are orthogonal, thus:

$$
\boldsymbol{I}^{2}=\boldsymbol{I}_{a}^{2}+\boldsymbol{I}_{r}^{2}+\boldsymbol{I}_{v}^{2}=\underbrace{\boldsymbol{I}_{a}^{b^{2}}+\boldsymbol{I}_{r}^{b^{2}}}_{\boldsymbol{I}^{b 2}}+\underbrace{\boldsymbol{I}_{a}^{u^{2}}+\boldsymbol{I}_{r}^{u^{2}}}_{\boldsymbol{I}^{u 2}}+\boldsymbol{I}_{v}^{2}
$$

From (8), we decompose the apparent power $A$ as:

$$
A=\mathbf{U} \mathbf{I}=\sqrt{P^{2}+Q^{2}+N_{a}^{2}+N_{r}^{2}+D^{2}}
$$

where:

$$
\begin{aligned}
& \text { - } P=\mathbf{U} \mathbf{I}_{a}^{b} \quad \text { Active Power } \\
& \text { - } Q=\mathbf{U} \mathbf{I}_{r}^{b} \quad \text { Reactive Power } \\
& \text { - } N_{a}=\mathbf{U} \mathbf{I}_{a}^{u} \quad \text { Unbalance Active Power } \\
& \text { - } N_{r}=\mathbf{U} \mathbf{I}_{r}^{u} \\
& \text { Unbalance Reactive Power (9.d) } \\
& \text { - } N=\sqrt{N_{a}^{2}+N_{r}^{2}} \quad \text { Unbalance Power } \\
& \text { - } D=\mathbf{U} \mathbf{I}_{v} \quad \text { Distortion Power }
\end{aligned}
$$

Note that all quantities (9) can be computed in the time domain. Moreover, (5.b) and (9.b) show that reactive power $Q$ and reactive energy $W$ are related by:

$$
Q=W \mathbf{U} / \hat{\mathbf{U}}
$$

For sinusoidal operation $\mathbf{U} / \widehat{\mathbf{U}}=\omega$ ( $\omega$ is angular line frequency), thus $Q=\omega W$. Apart from $P$, all power terms (9) characterize a non-ideal aspect of the load behavior. The global performance index is the power factor:

$$
\lambda=\frac{P}{A}=\frac{\boldsymbol{I}_{a}}{\boldsymbol{I}}=\frac{P}{\sqrt{P^{2}+Q^{2}+N_{a}^{2}+N_{r}^{2}+D^{2}}}
$$

It can be calculated for every circuit independently of waveform distortion and asymmetry, and is affected not only by load unbalance and non-linearity, but also by supply asymmetry and distortion.

The separation of supply and load responsibility is the goal of the accountability approach discussed in this paper, which makes use of load and supply modeling and sequence decomposition of voltages and currents

\section{LOAD MODELING}

The load modeling approach was firstly analyzed in [21] and is reconsidered here with reference to the general perphase equivalent circuit of Fig.1.a, which holds for a generic inductive load with positive reactive energy.

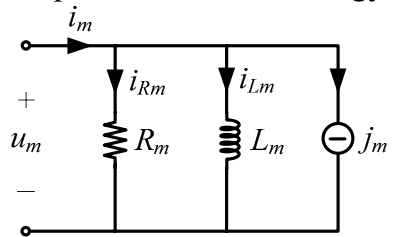

Fig. 1.a. Per-phase equivalent circuit of inductive load

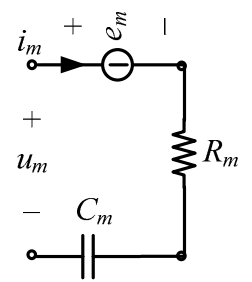

Fig. 1.b. Per-phase equivalent circuit of capacitive load

Let's assume that the measuring equipment at the load terminals (PCC) senses phase voltage $u_{m}$ and current $i_{m}$ and discriminates their fundamental $\left(u_{m}^{f}, i_{m}^{f}\right)$ and harmonic $\left(u_{m}^{h}, i_{m}^{h}\right)$ components.

Parameters $R_{m}$ and $L_{m}$ of the Fig.1.a are chosen to suit the circuit performance at fundamental frequency. They are derived from power and energy measurements as:

$$
\begin{array}{ll}
R_{m}=\frac{U_{m}^{f 2}}{P_{m}^{f}}, & P_{m}^{f}=\left\langle u_{m}^{f}, i_{m}^{f}\right\rangle \\
L_{m}=\frac{\hat{U}_{m}^{f 2}}{W_{m}^{f}}, & W_{m}^{f}=\left\langle\hat{u}_{m}^{f}, i_{m}^{f}\right\rangle
\end{array}
$$


Applying voltage $u_{m}$ to the equivalent circuit gives:

$$
\begin{aligned}
i_{m} & =\frac{u_{m}}{R_{m}}+\frac{\hat{u}_{m}}{L_{m}}+j_{m}= \\
& =\frac{u_{m}^{f}}{R_{m}}+\frac{\hat{u}_{m}^{f}}{L_{m}}+\frac{u_{m}^{h}}{R_{m}}+\frac{\hat{u}_{m}^{h}}{L_{m}}+j_{m}
\end{aligned}
$$

Since from definitions (12) we get:

$$
i_{m}^{f}=\frac{u_{m}^{f}}{R_{m}}+\frac{\hat{u}_{m}^{f}}{L_{m}}
$$

the current source $j_{m}$ can be expressed by difference as:

$$
j_{m}=i_{m}^{h}-\frac{u_{m}^{h}}{R_{m}^{f}}-\frac{\widehat{u}_{m}^{h}}{L_{m}^{f}}
$$

Thus, $j_{m}$ contains only harmonic terms, which are determined by supply distortion and load non-linearity.

If the load is capacitive, reactive energy $W_{m}^{f}$ becomes negative and the parallel connection of Fig.1.a can be replaced, by duality, by the series connection of Fig.1.b, the equivalent phase resistance $R_{m}$ and capacitor $C_{m}$ being:

$$
R_{m}=\frac{P_{m}^{f}}{I_{m}^{f 2}}, \quad C_{m}=-\frac{\hat{I}_{m}^{f 2}}{W_{m}^{f}}
$$

Voltage source $e_{m}$ contains only harmonics and is given by:

$$
e_{m}=u_{m}^{h}-R_{m} i_{m}^{h}-\frac{\hat{i}_{m}^{h}}{C_{m}}
$$

At fundamental frequency, the two models of Fig.1 are perfectly equivalent, of course.

This modeling approach requires that the equivalent circuit parameters keep almost the same even when supply voltages vary (within reasonable limits) in amplitude, phase, symmetry and distortion. This normally happens for line-toneutral loads, that are accurately modeled by the equivalent circuit of Fig.1, while it is only approximately true for lineto-line loads: in this case, in fact, the model can misbehave in presence of severe load unbalance. In practice, however, delta-connected loads fed by the distribution grid are nearly balanced, and do not affect the model validity. With these limits in mind, the model makes possible an accountability approach based only on measurements at the PCC, without knowing load structure nor supply parameters.

Consider also that smart grids are characterized by distributed active loads and sources, interfaced by electronic power converters, which often operate at constant power. Even in this case the equivalent circuits of Fig. 1 hold, where $R_{m}$ takes positive or negative values depending on the sign of measured active power.

The effect of voltage harmonics can be analyzed in differential terms (small-signal analysis around the "quiet" condition set by operation at fundamental frequency), under the assumption of limited supply distortion. In particular, for operation at constant power $\left(P_{m}=u_{m} i_{m}\right)$, the differential resistance $r_{m}$ becomes:

$$
r_{m}=\left[\frac{\partial u_{m}}{\partial i_{m}}\right]_{\text {quiet }}=\left[\frac{\partial}{\partial i_{m}} \frac{P_{m}}{i_{m}}\right]_{\text {quiet }}=-\frac{P_{m}}{I_{m}^{2}}=-R_{m}
$$

\section{Power Terms vs Sequence COMPONENTS}

Before entering the accountability problem, this section analyzes the sequence decomposition of active power and reactive energy at PCC, based on the proposed load model. This decomposition allows better understanding of the nonideal power terms generated at PCC, and will also be used for the accountability analysis.

The voltages and currents measured at PCC can be decomposed in their fundamental and harmonic terms, the fundamental components being further split into positive, negative, and zero sequence terms, i.e.:

$$
\begin{aligned}
& \mathbf{u}=\mathbf{u}^{f}+\mathbf{u}^{h}=\mathbf{u}^{p}+\mathbf{u}^{n}+\mathbf{u}^{z}+\mathbf{u}^{h} \\
& \mathbf{i}=\mathbf{i}^{f}+\mathbf{i}^{h}=\mathbf{i}^{p}+\mathbf{i}^{n}+\mathbf{i}^{z}+\mathbf{i}^{h}
\end{aligned}
$$

Sequence components are always orthogonal, thus the internal product of quantities of different sequence is zero. Harmonic terms are also orthogonal to fundamental ones, thus we may split the collective rms values of voltages and currents as:

$$
\begin{aligned}
& \mathbf{U}=\mathbf{U}^{f^{2}}+\mathbf{U}^{h^{2}}=\mathbf{U}^{p^{2}}+\mathbf{U}^{n^{2}}+\mathbf{U}^{z^{2}}+\mathbf{U}^{h^{2}} \\
& \mathbf{I}=\mathbf{I}^{f^{2}}+\mathbf{I}^{h^{2}}=\mathbf{I}^{p^{2}}+\mathbf{I}^{n^{2}}+\mathbf{I}^{z^{2}}+\mathbf{I}^{h^{2}}
\end{aligned}
$$

The power and energy terms associated to sequence and harmonic components are independent from each other and sum up to give the total amount. Thus, we may split the power and energy measured at PCC as:

$$
\begin{aligned}
P & =P^{p}+P^{n}+P^{z}+P^{h}= \\
& =\left\langle\mathbf{u}^{p}, \mathbf{i}^{p}\right\rangle+\left\langle\mathbf{u}^{n}, \mathbf{i}^{n}\right\rangle+\left\langle\mathbf{u}^{z}, \mathbf{i}^{z}\right\rangle+\left\langle\mathbf{u}^{h}, \mathbf{i}^{h}\right\rangle \\
W & =W^{p}+W^{n}+W^{z}+W^{h}= \\
& =\left\langle\widehat{\mathbf{u}}^{p}, \mathbf{i}^{p}\right\rangle+\left\langle\hat{\mathbf{u}}^{n}, \mathbf{i}^{n}\right\rangle+\left\langle\hat{\mathbf{u}}^{z}, \mathbf{i}^{z}\right\rangle+\left\langle\widehat{\mathbf{u}}^{h}, \mathbf{i}^{h}\right\rangle
\end{aligned}
$$

Since $P$ and $W$ link respectively to active currents $\boldsymbol{i}_{\boldsymbol{a}}$ and reactive currents $\boldsymbol{i}_{\boldsymbol{r}}$, equations (18) can be expressed as a function of sequence and harmonic components of $\boldsymbol{i}_{\boldsymbol{a}}$ and $\boldsymbol{i}_{\boldsymbol{r}}$ :

$$
\begin{aligned}
& P=\underbrace{\left\langle\mathbf{u}^{p}, \mathbf{i}_{a}^{p}\right\rangle}_{P^{p}}+\underbrace{\left\langle\mathbf{u}^{n}, \mathbf{i}_{a}^{n}\right\rangle}_{P^{n}}+\underbrace{\left\langle\mathbf{u}^{z}, \mathbf{i}_{a}^{z}\right\rangle}_{P^{z}}+\underbrace{\left\langle\mathbf{u}^{h}, \mathbf{i}_{a}^{h}\right\rangle}_{P^{h}} \\
& W=\underbrace{\left\langle\widehat{\mathbf{u}}^{p}, \mathbf{i}_{r}^{p}\right\rangle}_{W^{p}}+\underbrace{\left\langle\hat{\mathbf{u}}^{n}, \mathbf{i}_{r}^{n}\right\rangle}_{W^{n}}+\underbrace{\left\langle\widehat{\mathbf{u}}^{z}, \mathbf{i}_{r}^{z}\right\rangle}_{W^{z}}+\underbrace{\left\langle\hat{\mathbf{u}}^{h}, \mathbf{i}_{r}^{h}\right\rangle}_{W^{h}}
\end{aligned}
$$

Considering now the fundamental components only we can refer to the circuit of Fig.1.a, which is equivalent to that of Fig.1.b. We express the active and reactive currents as:

$$
\mathbf{i}_{a}^{f}=\mathbf{G u}^{f}, \mathbf{G}=\left|\begin{array}{ccc}
G_{1} & 0 & 0 \\
0 & G_{2} & 0 \\
0 & 0 & G_{3}
\end{array}\right|=\left|\begin{array}{ccc}
\frac{1}{R_{1}} & 0 & 0 \\
0 & \frac{1}{R_{2}} & 0 \\
0 & 0 & \frac{1}{R_{3}}
\end{array}\right|
$$




$$
\mathbf{i}_{r}^{f}=\mathbf{B} \widehat{\mathbf{u}}^{f}, \mathbf{B}=\left|\begin{array}{ccc}
B_{1} & 0 & 0 \\
0 & B_{2} & 0 \\
0 & 0 & B_{3}
\end{array}\right|=\left|\begin{array}{ccc}
\frac{1}{L_{1}} & 0 & 0 \\
0 & \frac{1}{L_{2}} & 0 \\
0 & 0 & \frac{1}{L_{3}}
\end{array}\right|
$$

The sequence components of the active currents can be derived in the time domain, according to [23], by:

$$
\begin{aligned}
& i_{a 1}^{p}(t)=\frac{1}{3}\left(i_{a 1}(t)+i_{a 2}\left(t-\frac{T}{3}\right)+i_{a 3}\left(t-\frac{2 T}{3}\right)\right) \\
& i_{a 1}^{n}(t)=\frac{1}{3}\left(i_{a 1}(t)+i_{a 2}\left(t+\frac{T}{3}\right)+i_{a 3}\left(t+\frac{2 T}{3}\right)\right) \\
& i_{a 1}^{z}(t)=\frac{1}{3}\left(i_{a 1}(t)+i_{a 2}(t)+i_{a 3}(t)\right)
\end{aligned}
$$

Only phase 1 is considered, the other phase quantities being immediately derived for each sequence.

From (20) and (21) we derive the sequence components of the active currents in the form:

$$
\begin{aligned}
& \mathbf{i}_{a}^{p}=\bar{G} \mathbf{u}^{p}+\mathbf{G}_{n}^{p} \mathbf{u}^{n}+\mathbf{G}_{z}^{p} \mathbf{u}_{\nabla}^{z} \\
& \mathbf{i}_{a}^{n}=\mathbf{G}_{p}^{n} \mathbf{u}^{p}+\bar{G} \mathbf{u}^{n}+\mathbf{G}_{z}^{n} \mathbf{u}_{\nabla}^{z} \\
& \mathbf{i}_{a}^{z}=\mathbf{G}_{p}^{z} \mathbf{u}^{p}+\mathbf{G}_{n}^{z} \mathbf{u}^{n}+\bar{G} \mathbf{u}^{z}
\end{aligned}
$$

where $u_{1}^{z}=u_{2}^{z}=u_{3}^{z}=u^{z}$, and

$$
\mathbf{u}_{\nabla}^{z}=\left|\begin{array}{c}
u^{z}(t) \\
u^{z}\left(t-\frac{T}{3}\right) \\
u^{z}\left(t+\frac{T}{3}\right)
\end{array}\right|
$$

Now, let:

$$
\bar{G}=\frac{G_{1}+G_{2}+G_{3}}{3},
$$

be the average (balanced) load conductance, and:

$$
\Delta G_{m}=G_{m}-\bar{G}
$$

be the unbalance conductance of phase $m(m=1 \ldots 3)$, the above conductance matrices can be expressed as:

$$
\begin{aligned}
& \mathbf{G}_{n}^{p}=\mathbf{G}_{p}^{n}=\frac{1}{3}\left|\begin{array}{lll}
\Delta G_{1} & \Delta G_{3} & \Delta G_{2} \\
\Delta G_{3} & \Delta G_{2} & \Delta G_{1} \\
\Delta G_{2} & \Delta G_{1} & \Delta G_{3}
\end{array}\right| \\
& \mathbf{G}_{z}^{p}=\frac{1}{3}\left|\begin{array}{lll}
\Delta G_{1} & \Delta G_{3} & \Delta G_{2} \\
\Delta G_{2} & \Delta G_{1} & \Delta G_{3} \\
\Delta G_{3} & \Delta G_{2} & \Delta G_{1}
\end{array}\right| \\
& \mathbf{G}_{z}^{n}=\frac{1}{3}\left|\begin{array}{lll}
\Delta G_{1} & \Delta G_{2} & \Delta G_{3} \\
\Delta G_{2} & \Delta G_{3} & \Delta G_{1} \\
\Delta G_{3} & \Delta G_{1} & \Delta G_{2}
\end{array}\right|
\end{aligned}
$$

$$
\mathbf{G}_{p}^{z}=\mathbf{G}_{n}^{z}=\frac{1}{3}\left|\begin{array}{lll}
\Delta G_{1} & \Delta G_{2} & \Delta G_{3} \\
\Delta G_{1} & \Delta G_{2} & \Delta G_{3} \\
\Delta G_{1} & \Delta G_{2} & \Delta G_{3}
\end{array}\right|
$$

Equations (22) and (25) show that positive-sequence voltages can generate negative and zero-sequence currents only in presence of load unbalance, and the same happens for the other sequence components. From (19) we derive the sequence power terms as:

$$
\begin{aligned}
& P^{p}=\underbrace{\left\langle\mathbf{u}^{p}, \bar{G} \mathbf{u}^{p}\right\rangle}_{P_{p}^{p}}+\underbrace{\left\langle\mathbf{u}^{p}, \mathbf{G}_{n}^{p} \mathbf{u}^{n}\right\rangle}_{P_{n}^{p}}+\underbrace{\left\langle\mathbf{u}^{p}, \mathbf{G}_{z}^{p} \mathbf{u}_{\nabla}^{z}\right\rangle}_{P_{z}^{p}} \\
& P^{n}=\underbrace{\left\langle\mathbf{u}^{n}, \mathbf{G}_{p}^{n} \mathbf{u}^{p}\right\rangle}_{P_{p}^{n}}+\underbrace{\left\langle\mathbf{u}^{n}, \bar{G} \mathbf{u}^{n}\right\rangle}_{P_{n}^{n}}+\underbrace{\left\langle\mathbf{u}^{n}, \mathbf{G}_{z}^{n} \mathbf{u}_{\nabla}^{z}\right\rangle}_{P_{z}^{n}} \\
& P^{z}=\underbrace{\left\langle\mathbf{u}^{z}, \mathbf{G}_{p}^{z} \mathbf{u}^{p}\right\rangle}_{P_{p}^{z}}+\underbrace{\left\langle\mathbf{u}^{n}, \mathbf{G}_{n}^{z} \mathbf{u}^{n}\right\rangle}_{P_{n}^{z}}+\underbrace{\left\langle\mathbf{u}^{z}, \bar{G} \mathbf{u}^{z}\right\rangle}_{P_{z}^{z}}
\end{aligned}
$$

It is easy to demonstrate that the sequence power terms are reciprocal, i.e.:

$$
P_{p}^{n}=P_{n}^{p}, \quad P_{n}^{z}=P_{z}^{n}, \quad P_{z}^{p}=P_{p}^{z}
$$

and this is true separately for each phase, i.e.:

$$
P_{p, m}^{n}=P_{n, m}^{p}, \quad P_{n, m}^{z}=P_{z, m}^{n}, \quad P_{z, m}^{p}=P_{p, m}^{z}
$$

In the usual condition where positive-sequence voltages are much higher than negative- and zero-sequence terms, we can order the above power terms for relevance as follows:

$$
\left.\left.\left.\left.\left|P_{p}^{p}\right|\right\rangle\right\rangle\left|P_{p}^{n}\right|,\left|P_{p}^{z}\right|\right\rangle\right\rangle\left|P_{n}^{n}\right|,\left|P_{n}^{z}\right|,\left|P_{z}^{z}\right|
$$

We can apply the same considerations to reactive current and energy terms. Thus, substituting in (22) phase reactivity $B_{m}=1 / L_{m}$ in place of conductance $G_{m}$, we get:

$$
\begin{aligned}
\mathbf{i}_{r}^{p} & =\bar{B} \widehat{\mathbf{u}}^{p}+\mathbf{B}_{n}^{p} \widehat{\mathbf{u}}^{n}+\mathbf{B}_{z}^{p} \widehat{\mathbf{u}}_{\nabla}^{z} \\
\mathbf{i}_{r}^{n} & =\mathbf{B}_{p}^{n} \widehat{\mathbf{u}}^{p}+\bar{B} \widehat{\mathbf{u}}^{n}+\mathbf{B}_{z}^{n} \widehat{\mathbf{u}}_{\nabla}^{z} \\
\mathbf{i}_{r}^{z} & =\mathbf{B}_{p}^{z} \widehat{\mathbf{u}}^{p}+\mathbf{B}_{n}^{z} \widehat{\mathbf{u}}^{n}+\bar{B} \widehat{\mathbf{u}}^{z}
\end{aligned}
$$

and:

$$
\begin{aligned}
& W^{p}=\left\langle\widehat{\mathbf{u}}^{p}, \mathbf{i}_{r}^{p}\right\rangle=W_{p}^{p}+W_{n}^{p}+W_{z}^{p} \\
& W^{n}=\left\langle\widehat{\mathbf{u}}^{n}, \mathbf{i}_{r}^{n}\right\rangle=W_{p}^{n}+W_{n}^{n}+W_{z}^{n} \\
& W^{z}=\left\langle\widehat{\mathbf{u}}^{z}, \mathbf{i}_{r}^{z}\right\rangle=W_{p}^{z}+W_{n}^{z}+W_{z}^{z}
\end{aligned}
$$

By ordering the reactive energy terms for relevance we get:

$$
\left.\left.\left.\left.\left|W_{p}^{p}\right|\right\rangle\right\rangle\left|W_{p}^{n}\right|,\left|W_{p}^{z}\right|\right\rangle\right\rangle\left|W_{n}^{n}\right|,\left|W_{n}^{z}\right|,\left|W_{z}^{z}\right|
$$

Obviously, in case of three-wire three-phase systems the zero-sequence components vanish in all equations.

In general, measures done at PCC cannot give enough information to identify the origin of unwanted power terms: in fact, asymmetric voltages or currents can be generated by either load unbalance or supply asymmetry. 


\section{EFFECTS OF SUPPly ASYMMETRY AND Distortion AND LINE IMPEDANCES}

In general, the voltages at PCC reflect any asymmetry and distortion of the source. They are also affected by load unbalance, which originates negative- and zero-sequence currents and voltage drops on line impedances. Similarly, harmonic currents absorbed by distorting loads flow in the supply lines and cause voltage distortion at PCC.

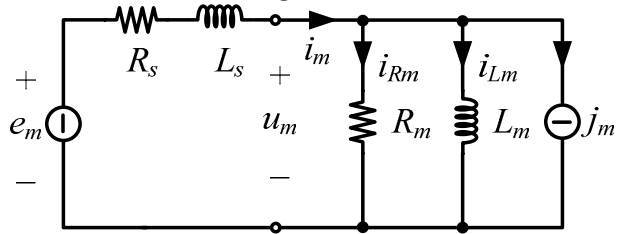

Fig. 2. Per-phase equivalent circuit including supply parameters

Consider the per-phase equivalent circuit of Fig.2, which represents the voltage supply by source voltage $e_{m}$ and line parameters $R_{S}$ and $L_{S}$. Assuming for symmetry that $R_{S}$ and $L_{S}$ are the same for all supply lines, the circuit equations for a generic poly-phase system become:

$$
\mathbf{e}=\mathbf{u}+R_{S} \mathbf{i}+L_{S} \frac{d \mathbf{i}}{d t}
$$

By splitting the voltages and currents in their sequence components, we get:

$$
\left\{\begin{array}{l}
\mathbf{e}^{p}=\mathbf{u}^{p}+R_{S} \mathbf{i}^{p}+L_{S} d \mathbf{i}^{p} / d t \\
\mathbf{e}^{n}=\mathbf{u}^{n}+R_{S} \mathbf{i}^{n}+L_{S} d \mathbf{i}^{n} / d t \\
\mathbf{e}^{z}=\mathbf{u}^{z}+R_{S} \mathbf{i}^{z}+L_{S} d \mathbf{i}^{z} / d t \\
\mathbf{e}^{h}=\mathbf{u}^{h}+R_{S} \mathbf{i}^{h}+L_{S} d \mathbf{i}^{h} / d t
\end{array}\right.
$$

Equations (32) show that, if the line parameters are known, we can easily derive the sequence and harmonic components of the source voltages from the measurements done at PCC. Thus, the load power absorption under purely sinusoidal and symmetrical supply can precisely be determined for accountability purposes.

If the supply circuit is more complex than that of Fig.2, e.g., due to the presence of compensation capacitors, the above approach can still be applied provided that the circuit model and equations are rearranged to suit the actual supply configuration.

Instead, if the line parameters are unknown the source voltages cannot be identified and the power terms measured at PCC cannot precisely be depurated from the effects of supply non-ideality and line impedances. Even in this case, however, the measurements at PCC allow a good estimation of actual power terms accountable to the load, as it will be shown in the next section.

As concerns the impact of the line impedance, note that the active power absorbed by $R_{S}$ is:

$$
P_{R s}=R_{S}\left(\mathbf{I}^{p^{2}}+\mathbf{I}^{n^{2}}+\mathbf{I}^{z^{2}}+\mathbf{I}^{h^{2}}\right)
$$

Thus, under ideal supply conditions $\left(\mathbf{e}=\mathbf{e}^{p}\right)$, the power terms measured at PCC would be:

$$
\begin{aligned}
& P^{p}=\left\langle e^{p}, i^{p}\right\rangle-R_{S} \mathbf{I}^{p^{2}} \\
& P^{n}=-R_{S} \mathbf{I}^{n^{2}}, \quad P^{z}=-R_{S} \mathbf{I}^{z^{2}}, \quad P^{h}=-R_{S} \mathbf{I}^{h^{2}}
\end{aligned}
$$

Since power terms $P^{n}, P^{z}$ and $P^{h}$ are negative, they reduce the active power measured at PCC, causing some under-penalization of the load. This effect, however, is due to line impedances, which are not under the responsibility of the load, and should not be considered as a factor for load penalization. The same happens for reactive energy terms.

\section{ACCOUNTABILITY}

Following the above approach we may estimate the load and supply contribution to the various power terms. The description reported here is limited to inductive models, but the same approach can be applied model including capacitive components.

Let's first assume that the line impedances are known. In this case we proceed as follows:

1) From the voltages and currents measured at PCC we estimate the parameters of the equivalent circuit of Fig.1, according to (12), (13), (14).

2) Given the supply line parameters $R_{S}$ and $L_{S}$, we determine the fundamental positive-sequence supply voltages $\boldsymbol{e}^{p}$ from (32.b).

3) Applying now supply voltages $\boldsymbol{e}^{p}$ to the equivalent circuit of Fig.3 we may estimate the fundamental phase currents $\mathbf{i}_{\ell}^{f}$ absorbed by the load under the ideal supply condition and the corresponding fundamental phase voltages $\mathbf{u}_{\ell}^{f}$ appearing at PCC. Any asymmetry of currents $\mathbf{i}_{\ell}^{f}$ and voltages $\mathbf{u}_{\ell}^{f}$ must be accounted to load unbalance, since supply voltages and line impedances are symmetrical.

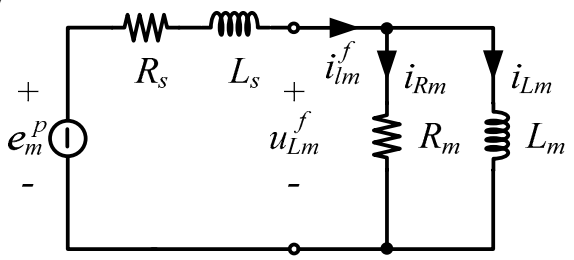

Fig. 3. Equivalent circuit to estimate fundamental voltages and currents appearing at PCC under ideal supply condition.

4) Then, applying harmonic current sources $\boldsymbol{j}$ acting in the load phases we may determine the harmonic voltages $\mathbf{u}_{\ell}^{h}$ and currents $\mathbf{i}_{\ell}^{h}$ occurring at PCC, which are also accountable to the load. Since line impedances are usually much smaller than load impedances we may refer to the simplified circuit of Fig. 4, thus:

$\mathbf{i}_{\ell}^{h} \cong \mathbf{j}$

$\mathbf{u}_{\ell}^{h} \cong-R_{S} \mathbf{j}-L_{S} \frac{d \mathbf{j}}{d t}$ 


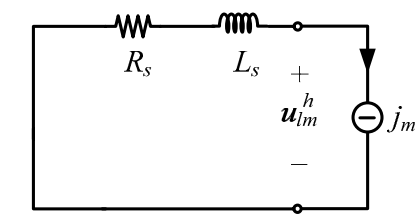

Fig. 4. Equivalent circuit to estimate harmonic voltages and currents at PCC due to load distortion.

5) Finally, we estimate the power terms (9) accountable to the load by considering the voltages and currents at PCC occurring for ideal supply conditions:

$$
\begin{gathered}
\boldsymbol{u}_{\ell}=\boldsymbol{u}_{\ell}^{f}+\boldsymbol{u}_{\ell}^{h} \\
\boldsymbol{i}_{\ell}=\boldsymbol{i}_{\ell}^{f}+\boldsymbol{i}_{\ell}^{h}
\end{gathered}
$$

Consider now the case where the line impedances are unknown. In theory, we may estimate the active power and reactive energy accountable to the load as the quantities $P_{p}^{p}$ and $W_{p}^{p}$ given by (26.a) and (30.a). In practice the estimation procedure may be affected by inaccuracy in both steps of separating positive-sequence terms and computing equivalent phase parameters. In particular, decomposing phase voltages may be difficult, since negative- and zerosequence terms can be much smaller than positive-sequence components. However, we can simplify the estimation of term $P_{p}^{p}$ by recalling inequalities (28). Observe in fact from (26) and (27) that:

$$
P^{p}-P^{n}-P^{z}=P_{p}^{p}+\underbrace{P_{n}^{n}+P_{z}^{z}+2 P_{n}^{z}}_{\text {very small }} \approx P_{p}^{p}
$$

and, similarly:

$$
W^{p}-W^{n}-W^{z} \approx W_{p}^{p}
$$

Moreover, sequence terms are orthogonal, thus:

$$
\begin{aligned}
& P^{p}=\left\langle\mathbf{u}, \mathbf{i}^{p}\right\rangle, \quad P^{n}=\left\langle\mathbf{u}, \mathbf{i}^{n}\right\rangle, \quad P^{z}=\left\langle\mathbf{u}, \mathbf{i}^{z}\right\rangle \\
& W^{p}=\left\langle\widehat{\mathbf{u}}, \mathbf{i}^{p}\right\rangle, \quad W^{n}=\left\langle\widehat{\mathbf{u}}, \mathbf{i}^{n}\right\rangle, \quad W^{z}=\left\langle\widehat{\mathbf{u}}, \mathbf{i}^{z}\right\rangle
\end{aligned}
$$

Estimation of $P_{p}^{p}$ and $W_{p}^{p}$ by (37) and (38) is easy to implement and only requires to determine the sequence components of the currents according to (21).

The accuracy of estimation provided by (37) is indeed very good, see Figs. 5 and 6 which refer to the application example of Fig. 8.

In particular, Fig.5 shows the estimation error due to (37.a) as a function of the amplitude and phase of the negative-sequence component of the source voltages $\left(\mathbf{e}^{n}\right)$. The line impedance is assumed to cause $5 \%$ voltage drop at the fundamental frequency, which is a realistic figure for low-voltage distribution lines. The estimation error is less than $0.1 \%$ in case of ideal supply $\left(\mathbf{e}^{n}=0\right)$ and remains below $0.5 \%$ even if negative-sequence voltages $\mathrm{e}^{n}$ increase up to $5 \%$ of positive-sequence voltages $\mathbf{e}^{p}$.

Similarly, Fig.6 shows the estimation error due to (37.a) as a function of the amplitude and phase of the zero- sequence source voltages $\left(\mathbf{e}^{z}\right)$. The same figure, for the sake of comparison, shows also the estimation error made by accounting the load for total active power measured at PCC. While the error caused by (37.a) remains well below $1 \%$, attributing the measured active power to the load can cause an over- or under-penalization up to $6 \%$, which is excessive for revenue metering. This demonstrates, on one side, the effectiveness of the proposed approach to identify power term $P_{p}^{p}$ accountable to the load and, on the other side, the need to revise the usual accounting procedures, which can severely over- or under-penalize the load.

Since similar results hold for $W_{p}^{p}$, we can conclude that the proposed accountability approach works generally well also when the line impedance is not known.

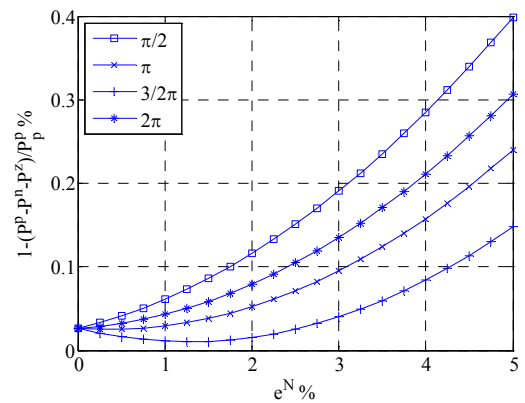

Fig. 5 Estimation error caused by (37.a) as a function of amplitude and phase of the negative sequence in source voltage

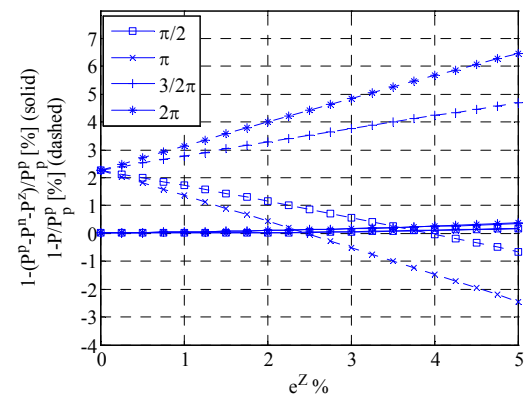

Fig. 6 Estimation error caused by (37.a) as a function of amplitude and phase of the zero sequence in supply voltage (solid line), compared with the error caused by direct application of (18.a) (dashed line)

\section{APPLICATION EXAMPLES}

The proposed load characterization and accountability approach has been extensively verified through simulation in Matlab-Simulink environment with PLECS toolbox. To give a concise representation of the results, only two relevant cases are reported here, referred to the loads of Figs.7 and 8. Without loss of generality, the considered loads are three phase low-voltage loads, with rated apparent power $A_{1}=18 \mathrm{kVA}$ for case 1 (Fig.7) and $\mathrm{A}_{2}=25 \mathrm{kVA}$ for case 2 (Fig.8). The supply voltages and line impedances are the same in both cases: the positive-sequence rms voltage (line to neutral) is $\mathrm{V}_{\mathrm{LN}}=230 \mathrm{~V}$ at $50 \mathrm{~Hz}$, while line impedance $\mathrm{Z}_{\mathrm{S}}$ is assumed to be $\mathrm{R}_{\mathrm{S}}-\mathrm{L}_{\mathrm{S}}$ (symmetric for the three phases), with $R_{S}=200 \mathrm{~m} \Omega$ and $L_{S}=200 \mu \mathrm{H}$. These values are characteristic of low voltage distribution cables, sized to 
give a voltage drop of 0.03 p.u. at maximum power (corresponding to Case 2).

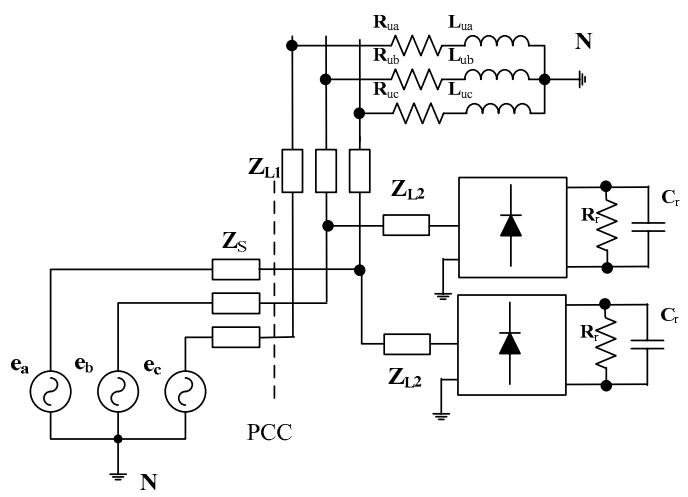

Fig. 7. Case 1: nonlinear unbalanced load

TABLE I. Load 1 parameters

\begin{tabular}{||c|c|}
\hline \hline $\mathrm{R}_{\mathrm{ua}}=20 \Omega \mathrm{R}_{\mathrm{ub}}=0.5 \Omega \mathrm{R}_{\mathrm{uc}}=20 \Omega$ & $\mathrm{R}_{\mathrm{r}}=25 \Omega$ \\
\hline $\mathrm{L}_{\mathrm{ua}}=40 \mathrm{mH} \mathrm{L}_{\mathrm{ub}}=20 \mathrm{mH}$ & $\mathrm{C}_{\mathrm{r}}=500 \mu \mathrm{F}$ \\
$\mathrm{L}_{\mathrm{uc}}=20 \mathrm{mH}$ & $\mathrm{Z}_{\mathrm{L} 2}=0.08+\mathrm{j} \omega 0.12 \mathrm{e}-3 \Omega$ \\
\hline $\mathrm{Z}_{\mathrm{L} 1}=0.1+\mathrm{j} \omega 0.2 \mathrm{e}-3 \Omega$ & \\
\hline
\end{tabular}

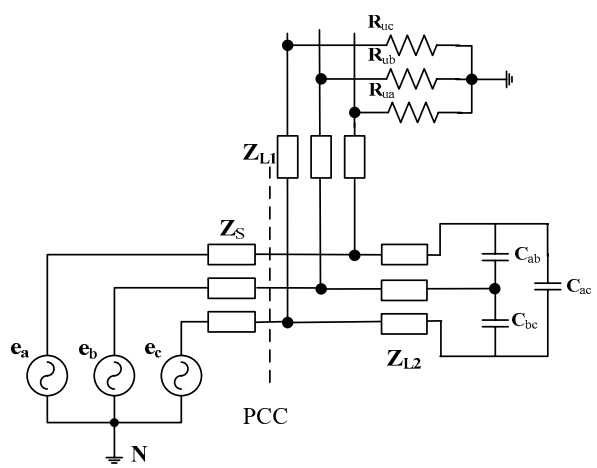

Fig. 8. Case 2: linear unbalanced load

TABLE II. Load 2 parameters.

\begin{tabular}{|c|c||}
\hline $\mathrm{R}_{\mathrm{ua}}=100 \Omega \mathrm{R}_{\mathrm{ub}}=10 \Omega \mathrm{R}_{\mathrm{uc}}=5 \Omega$ & $\mathrm{C}_{\mathrm{ab}}=\mathrm{C}_{\mathrm{bc}}=\mathrm{C}_{\mathrm{ac}}=100 \mu \mathrm{F}$ \\
\hline $\mathrm{Z}_{\mathrm{L} 1}=0.1+\mathrm{j} \omega 0.2 \mathrm{e}-3 \Omega$ & $\mathrm{Z}_{\mathrm{L} 2}=0.08+\mathrm{j} \omega 0.12 \mathrm{e}-3 \Omega$ \\
\hline
\end{tabular}

Tables I and II show the relevant load parameters.

In general, the load model discussed in the previous sections provides good accuracy only in case of linear wyeconnected loads. Distorting loads or delta-connected loads in four-wire systems can reduce the reliability of the model. The case studies were selected to test the proposed approach in critical situations. Case 1 investigates the effects of wyeconnected distorting loads, while Case 2 refers to balanced delta-connected load (e.g., a three-phase motor).

The simulation results are organized as follows: in both cases, power terms (9) are firstly computed from the direct measurements at the PCC, assuming the neutral wire as reference for voltage measurements. Then, following the accountability approach, the load model is derived and used to estimate the power terms accountable to the load. If the line impedances are known, the positive-sequence terms of the supply voltages are computed according to (32) and then applied to the model of Fig.2. If the line impedances are not known, the accountability approach is simplified by directly applying the positive-sequence components of the measured PCC voltages to the load model of Fig.1; alternatively, to avoid the decomposition of PCC voltages into sequence components, the approximation (37) can be applied.

The analysis is repeated for various types of non-ideality superimposed to the supply voltages: negative- and zerosequence terms (with variable amplitude and phase with respect to positive-sequence terms) and distortion.

Since the accountability procedure aims at depurating the power measurements from the terms due to supply nonideality, the data estimated with supply and load models are systematically compared with the actual data, obtained by supplying the actual load with positive-sequence sinusoidal voltages.

\section{A. Case 1: Nonlinear unbalanced load}

In this case, the load circuit includes wye-connected R-L loads and single-phase rectifiers with capacitive filters. According to the above modeling procedure, the harmonic currents absorbed by the load are represented by current sources. Accordingly, at fundamental frequency the model of Fig. 2 reflects accurately the behavior of actual system, provided that the line parameters are properly identified.

Table III gives the power terms (9) absorbed at PCC by the actual load if the source voltages are sinusoidal and symmetrical. They identify the power terms accountable to the load and represent the reference values that an ideal accountability approach should reproduce.

Table IV shows the amounts of power absorbed at PCC by the actual load under non-ideal voltage supply (PCC column), and the values estimated by the accountability method when the line impedance is known (LMZ column) or unknown (LM column). The data of Table IV are normalized to the corresponding values of Table 3, thus the performance of an accountability method is as better as closer it approaches unity value in all rows.

In particular, Table IV shows the estimation errors caused by various defects superimposed to source voltages: A) negative-sequence terms ( $5 \%$ of positive-sequence voltages and phase shift $\pi / 2)$; B) zero-sequence terms (5\% of positive-sequence voltages and phase shift $\pi / 2)$; C) distortion voltages $\left(5 \%\right.$ of $5^{\text {th }}$ harmonic, $2.5 \%$ of $7^{\text {th }}$ and $9^{\text {th }}$ harmonics).

The normalized data show that model LMZ properly estimates the active, reactive and unbalance power accountable to the load in cases A) and B), while estimation is less accurate in case $\mathrm{C}$ ). The difference between the actual power measured at PCC and that accounted to the load (using LMZ or LM methods) is non-negligible, in the order of few $\%$, thus justifying the modeling effort. In general, distortion power is estimated with less accuracy in all cases, and remains close to the actual values measured at PCC. This is a consequence of representing the nonlinear effects of the load by current sources, which do not reflect the actual behaviour of the load when subject to source voltage 
degradation. Finally, observe that in Case 1 the LM model behaves similarly to the LMZ model for every supply condition. Nevertheless, the quantities estimated by LMZ are slightly closer to the ideal values.

TABLE III - Power terms accountable to the load in Case 1, i.e., power measured at load terminals for sinusoidal and symmetrical supply

\begin{tabular}{|l|c|}
\hline \hline $\mathbf{P}_{\mathbf{1}}[\mathbf{k W}]$ & 10.810 \\
\hline $\mathbf{Q}_{\mathbf{1}}[\mathbf{k V A R}]$ & 7.086 \\
\hline $\mathbf{N}_{\mathbf{1}}[\mathbf{k V A}]$ & 9.654 \\
\hline $\mathbf{D}_{\mathbf{1}}[\mathbf{k V A}]$ & 8.976 \\
\hline \hline
\end{tabular}

TABLE IV - Case 1: Power terms measured at load terminals (PCC) and estimated according to different accountability approaches: with line impedances known (LMZ) and unknown (LM)

\begin{tabular}{|c|c|c|c|}
\hline \multicolumn{4}{|c|}{ A) Non-ideal voltage components: $e^{n}=5 \% \varphi^{n}=\pi / 2$} \\
\hline Normalized power terms & PCC & LMZ & $\mathbf{L M}$ \\
\hline $\mathbf{P}_{\mathbf{N}}$ & 1.0129 & 0.9989 & 1.0055 \\
\hline $\mathbf{Q}_{\mathrm{N}}$ & 1.0744 & 0.9998 & 1.0064 \\
\hline $\mathbf{N}_{\mathbf{N}}$ & 1.0204 & 0.9996 & 1.0024 \\
\hline $\mathbf{D}_{\mathrm{N}}$ & 1.0237 & 1.0227 & 1.0212 \\
\hline \multicolumn{4}{|c|}{ B) Non-ideal voltage components: $e^{z}=5 \% \varphi^{z}=\pi / 2$} \\
\hline Normalized power terms & PCC & LMZ & $\mathbf{L M}$ \\
\hline $\mathbf{P}_{\mathbf{N}}$ & 0.9921 & 0.9994 & 1.0058 \\
\hline $\mathbf{Q}_{\mathbf{N}}$ & 0.9307 & 1.0001 & 1.0067 \\
\hline $\mathbf{N}_{\mathbf{N}}$ & 0.9811 & 0.9998 & 1.0028 \\
\hline $\mathbf{D}_{\mathrm{N}}$ & 0.9808 & 0.9794 & 0.9783 \\
\hline \multicolumn{4}{|c|}{$\begin{array}{l}\text { C) Non-ideal voltage components: } 5^{\text {th }}=5 \% 7^{\text {th }}=9^{\text {th }}=2.5 \% \\
\varphi=0\end{array}$} \\
\hline Normalized power terms & PCC & LMZ & $\mathbf{L M}$ \\
\hline $\mathbf{P}_{\mathbf{N}}$ & 1.0414 & 1.0202 & 1.0313 \\
\hline $\mathbf{Q}_{\mathrm{N}}$ & 0.9739 & 0.9788 & 0.9863 \\
\hline $\mathbf{N}_{\mathbf{N}}$ & 0.9504 & 0.9509 & 0.9553 \\
\hline $\mathbf{D}_{\mathrm{N}}$ & 1.3442 & 1.3417 & 1.3400 \\
\hline
\end{tabular}

\section{B. Case 2: Linear unbalanced load}

In this case the load is the combination of three wyeconnected unbalanced resistive loads and three deltaconnected balanced capacitors. The delta connection of the capacitors can potentially cause errors in load modelling and propagate them into the accountability process. In practice, we verified that the effect of balanced three-wires loads is limited, as confirmed also by the results of Tables V and VI. These tables show normalized data and refer to two types of disturbances superimposed to positive-sequence supply voltages: A) negative-sequence voltages (amplitude 5\%, phase $\pi$; B) zero-sequence voltages (amplitude 5\%, phase $\pi$ ). The estimation done by LMZ gives, also in this case, the best results. However, compared to Case 1, the difference between LMZ and LM is more evident: in fact, LM is overestimating the active power by almost $2 \%$. This can be attributed to the higher power rating, which magnifies the effect of line impedances on the measurements at PCC.

TABLE V - Power terms accountable to the load in Case 2, i.e., power measured at load terminals for sinusoidal and symmetrical supply

\begin{tabular}{|l|c|}
\hline \hline $\mathbf{P}_{2}[\mathrm{~kW}]$ & 15.456 \\
\hline $\mathbf{Q}_{2}[\mathbf{k V A R}]$ & -14.511 \\
\hline $\mathbf{N}_{2}[\mathbf{k V A}]$ & 11.668 \\
\hline $\mathbf{D}_{2}[\mathbf{k V A}]$ & 0 \\
\hline \hline
\end{tabular}

TABLE VI - Case 2: Power terms measured at load terminals (PCC) and estimated according to different accountability approaches: with line impedances known (LMZ) and unknown (LM)

\begin{tabular}{|l||c|c|c||}
\hline \multicolumn{4}{|l||}{ A) Non-ideal voltage components: $\mathrm{e}^{\mathrm{n}}=5 \% \varphi^{\mathrm{n}}=\pi$} \\
\hline Normalized power terms & PCC & $\mathbf{L M Z}$ & $\mathbf{L M}$ \\
\hline $\mathbf{P}_{\mathbf{N}}$ & 1.0469 & 0.9988 & 1.0199 \\
\hline $\mathbf{Q}_{\mathbf{N}}$ & 1.0003 & 0.9996 & 0.9968 \\
\hline $\mathbf{N}_{\mathbf{N}}$ & 0.9887 & 1.0016 & 0.9987 \\
\hline $\mathbf{D}_{\mathbf{N}}$ & 0 & 0 & 0 \\
\hline
\end{tabular}

B) Non-ideal voltage components: $e^{z}=5 \% \varphi^{z}=\pi$

\begin{tabular}{|l||c|c|c||}
\hline Normalized power terms & PCC & LMZ & LM \\
\hline $\mathbf{P}_{\mathbf{N}}$ & 1.0461 & 0.9980 & 1.0207 \\
\hline $\mathbf{Q}_{\mathbf{N}}$ & 0.9989 & 1.0008 & 0.9973 \\
\hline $\mathbf{N}_{\mathbf{N}}$ & 0.9989 & 1.0008 & 0.9973 \\
\hline $\mathbf{D}_{\mathbf{N}}$ & 0 & 0 & 0 \\
\hline
\end{tabular}

C. Case 3: RLC balanced load

As a final example to show the performances and limits of the approach in case of loads with capacitive and inductive elements, an RLC wye-connected load was considered. The three-phase four-wire load includes a parallel connection of $R=0.5 \Omega, L=1.59 \mathrm{mH}$ and $C=3.29 \mathrm{mF}$ in each phase. The rms line-to-neutral voltage is $\mathrm{V}_{\mathrm{LN}}=127 \mathrm{~V}$ at $50 \mathrm{~Hz}$, and the line impedance is reduced to $1 / 20$ of previous value to meet the higher load power with the same voltage drop at PCC $(0.03$ p.u.). The line voltage is distorted by a positive-sequence $5^{\text {th }}$ harmonic, in phase with the fundamental term and with rms value $\mathrm{V}_{\mathrm{LN} 5}=6.35 \mathrm{~V}$. The results are shown in Table VIII, where the active and reactive power are normalized to the values of Table VII, while unbalance and distortion power are given in real values.

TABLE VII - Power terms accountable to the load in Case 3, i.e., power measured at load terminals for sinusoidal and symmetrical supply

\begin{tabular}{|l|c|}
\hline \hline $\mathbf{P}_{\mathbf{3}}[\mathbf{k W}]$ & 92.40 \\
\hline $\mathbf{Q}_{3}$ [kVAR] & 44.61 \\
\hline $\mathbf{N}_{\mathbf{3}}$ [kVA] & 0 \\
\hline $\mathbf{D}_{\mathbf{3}}$ [kVA] & 0 \\
\hline \hline
\end{tabular}

TABLE VIII - Case 3: Power terms measured at load terminals (PCC) and estimated according to different accountability approaches: with line impedances known (LMZ) and unknown (LM)

\begin{tabular}{|l||c|c|c||}
\hline \multicolumn{4}{|l||}{ Non ideal voltage components: $5^{\text {th }}=6.35 \mathrm{~V}(\mathrm{RMS}), \varphi=0$} \\
\hline Power terms & PCC & LMZ & LM \\
\hline $\mathbf{P}_{\mathbf{N}}$ (normalized) & 1.0029 & 0.9999 & 0.9988 \\
\hline $\mathbf{Q}_{\mathbf{N}}$ (normalized) & 0.9972 & 1.0003 & 0.9988 \\
\hline N [kVA] & 0 & 0 & 0 \\
\hline D [kVA] & 12.292 & 12.276 & 12.258 \\
\hline
\end{tabular}

The table shows how the proposed approach behaves in the specific operating condition. The estimated active power is slightly lower than that measured at PCC, since the 
algorithm tends to correct the effect of supply distortion. For similar reasons the estimated reactive power is slightly higher than that measured at PCC. In fact, the algorithm tends to correct the increase of capacitive power absorption caused by voltage distortion. Note also that the estimated distortion power is very close to the value measured at PCC, showing that the algorithm fails to discriminate the power terms due to supply distortion from those caused by load harmonics. In this case, the estimation algorithm provides the same accuracy of the measurement at PCC.

In conclusion, we can say that the proposed accountability approach works generally well and is capable to depurate the power accounted to the loads from the effects generated by supply deterioration. The estimation fails to give accurate results only when unbalanced delta-connected loads are fed by four-wire supply systems. The estimation of distortion power can also be affected by a certain level of inaccuracy, however over-penalization of the load is prevented.

\section{CONCLUSIONS}

In this paper the Conservative Power Theory (CPT) has been applied to face the problem of load characterization and revenue metering.

The proposed approach brings to a general method to separate load and supply responsibility on the generation of active, reactive, unbalance and distortion power.

The method relies on load and supply models derived by voltage and current measurements at PCC. It can be applied to revenue metering since it allows an accurate estimation of the power accountable to the load by depurating the effects of line impedances and source voltage deterioration on the measurements done at the point of common coupling.

The accountability procedure was tested extensively in a variety of applications, and demonstrated good accuracy even in presence of large variations of supply and load configuration and parameters.

The critical situations where the method can fail to provide accurate estimation of the various power terms were also identified and discussed. It was verified that estimation accuracy is never worse than that of direct measurements at the point of common coupling, thus over-penalization of the loads is prevented.

\section{REFERENCES}

[1] A. Ferrero, L. Peretto and R. Sasdelli, "Revenue metering in the presence of distortion and unbalance: myths and reality", in Proc. of IEEE International Conference on Harmonics and Quality of Power, pp. 42-47, 1998.

[2] R. Sasdelli, C. Muscas and L. Peretto, "A VI-Based Measurement System for Sharing the Customer and Supply Responsibility for Harmonic Distortion", IEEE Trans. on Instrumentation and Measurement, vol. 47, no. 5, pp. 1335-1340, October 1998.

[3] D.P. Manjure and E.B. Makram, "Effect of Nonlinearity and Unbalance on Power Factor," in Proc. of IEEE Power Engineering Society Summer Meeting, pp. 956-962, 2000.

[4] A. Pavas, V. Staudt, H. Torres-Sánchez, "Discussion on existing methodologies for the resposabilities assigment problem", International School on Nonsinusoidal Currents and Compensation, Lagow, Poland, June 2008.
[5] A. Ferrero, M. Faifer and S. Salicone, "On Testing the Electronic Revenue Energy Meters", IEEE Trans. on Instrumentation and Measurement, vol. 58, no. 9, pp. 3042-3049, September 2009.

[6] L. Cristaldi, A. Ferrero, "A digital method for the identification of the source of distortion in electric power systems", IEEE Trans. on Instrumentation and Measurement, vol. 44, no. 1, pp. 14-18, February 1994.

[7] W. Xu, X. Liu and Y. Liu, "An Investigation on the Validity of Power-Direction Method for Harmonic Source Determination", IEEE Trans. on Power Delivery, vol. 18, no. 1, pp. 214-219, January 2003.

[8] N. Locci, C. Muscas and S. Sulis, "Investigation on the Accuracy of Harmonic Pollution Metering Techniques", IEEE Trans. on Instrumentation and Measurement, vol. 53, no. 4, pp. 1140-1145, August 2004.

[9] G. D'Antona, C. Muscas, P. A. Pegoraro and S. Sulis, "Harmonic Source Estimation in Distribution Systems" IEEE Trans. on Instrumentation and Measurement, vol. 60, no. 10, pp. 3351-3359, October 2011.

[10] C. I. Budeanu, "Puissances reactives et fictives", Institute Romain de 1.Energie, Bucharest, 1927.

[11] S. Fryze, "Wirk-, Blind-,Scheinleistung in Elektrische Stromkreisen mit nichtsinusformingen Verlauf von Strom und Spannung", ETZ, Bd. 53, pp. 596-599, 625-627, 700-702, 1932.

[12] M. Depenbrock, "The FBD-Method, a Generally Applicable Tool for Analyzing Power Relations", IEEE Trans. on Power Systems, vol. 8, no 2, pp. 381-387, May 1993.

[13] J. L. Willems, J. L., "Instantaneous sinusoidal and harmonic active and deactive currents in three-phase power systems", European Trans. on Electrical Power Engineering, 4(5), pp. 335-343, 1994.

[14] L. Cristaldi, A. Ferrero, "Mathematical foundations of the instantaneous power concept: an algebraic approach", European Trans. on Electrical Power, vol. 6, no. 5, pp. 305-309, Sept./Oct. 1996.

[15] L. S. Czarnecki, "Currents' Physical Components (CPC) Concept: a Fundamental of Power Theory", Przegląd Elektrotechniczny no. 6, pp. 28-37, 2008.

[16] IEEE Trial-Use Standard Definitions for the Measurement of Electric Power Quantities under Sinusoidal, Non-sinusoidal, Balanced or Unbalanced Conditions, IEEE Standard 1459, 2010.

[17] J. L. Willems, "Reflections on Power Theories for Poly-Phase Nonsinusoidal Voltages and Currents”, Przegląd Elektrotechniczny no. 6, pp. 11-21, 2010.

[18] P. Tenti, P. Mattavelli, H. K. Morales P, "Conservative Power Theory, Sequence Components and Accountability in Smart Grids" Przeglad Elektrotechniczny, Vol. 6, pp. 30-37, 2010.

[19] P. Tenti, H. K. Morales P, P. Mattavelli, "Conservative Power Theory, a Framework to Approach Control and Accountability Issues in Smart Microgrids", IEEE Trans. on Power Electronics, vol. 26, no 3, pp. 664 - 673, March 2011.

[20] F. P. Marafao, E. V. Liberado, H. K. M. Paredes, L. C. P. da Silva, "Three-Phase Four-Wire Circuits Interpretation by means of Different Power Theories", Przegląd Elektrotechniczny no. 1, pp. 28-33, 2011.

[21] P. Tenti, H. K. M. Paredes, F. P. Marafão, P. Mattavelli, "Accountability in Smart Microgrids Based on Conservative Power Theory", IEEE Trans. on Instrumentation and Measurement, vol. 60, no 9, pp. 3058 - 3069, September 2011.

[22] H. K. M. Paredes, F. P. Marafão, P. Mattavelli and P. Tenti, "Application of Conservative Power Theory to Load and Line Characterization and Revenue Metering", Proc. of the IEEE International Workshop on Applied Measurements for Power Systems, pp. 1-6, 2012.

[23] P.Tenti, J.L.Willems, P.Mattavelli, E.Tedeschi, “Generalized symmetrical components for periodic non-sinusoidal three-phase signals", J. On Electrical Power Quality and Utilization, vol. XIII, n.1, p.9-15, 2007. 